\title{
Breast Metastasis in a Patient with Primary Hepatocellular Carcinoma
}

\author{
Bouchagier Konstantinos*, Solakis Evangelos, Fradelos Evangelos and Stylianidis Georgios \\ $2^{\text {nd }}$ Surgical Department, Evangelismos General Hospital of Athens, Greece
}

*Corresponding author: Bouchagier Konstantinos, General Surgeon, $2^{\text {nd }}$ Surgical department, Evangelismos General Hospital of Athens, Greece.

Received Date: August 20, 2019

Published Date: September 06, 2019

\begin{abstract}
Hepatocellular carcinoma is the most common primary carcinoma of the liver, that metastasises most commonly to lungs, lymph nodes, bones and adrenal glands. We report a case of a 70 years old female, with a left breast metastasis from hepatocellular carcinoma, along with hepatocellular carcinoma recurrence in the liver, that underwent a left mastectomy. The pathological report confirmed the metastasis. To the best of our knowledge, this is the second study reporting a breast metastasis from primary hepatocellular carcinoma.

Keywords: Hepatocellular carcinoma; Breast metastasis
\end{abstract}

\section{Introduction}

Hepatocellular carcinoma (HCC), is the $4^{\text {th }}$ most common neoplasm in men and 9th most common neoplasm in women, making it one of the leading causes of cancer-related deaths (approximately 600.000 deaths/year worldwide) [1-12]. Its geographical distribution varies according to the prevalence of known aetiologic factors such as HBV, HCV infection, alcohol, cirrhosis [12]. Extrahepatic metastases occur in $14-37 \%$ of cases and are associated with poor prognosis (1 year survival<40\%)
$[10,13]$ The most common extrahepatic HCC metastases sites are the lungs (49\%), lymph nodes (41\%), bone metastases (16\%) and the adrenal glands (15\%) Metastases occur via the portal vein system or via mediastinal nodes [2]. Breast Ca is the most commonly diagnosed cancer in the world, among women, and the second leading cause of cancer deaths, after lung cancer [14]. Breast metastases are extremely rare, according to worldwide literature. This is the second report of breast metastasis to a patient with primary hepatocellular carcinoma.

Case Report
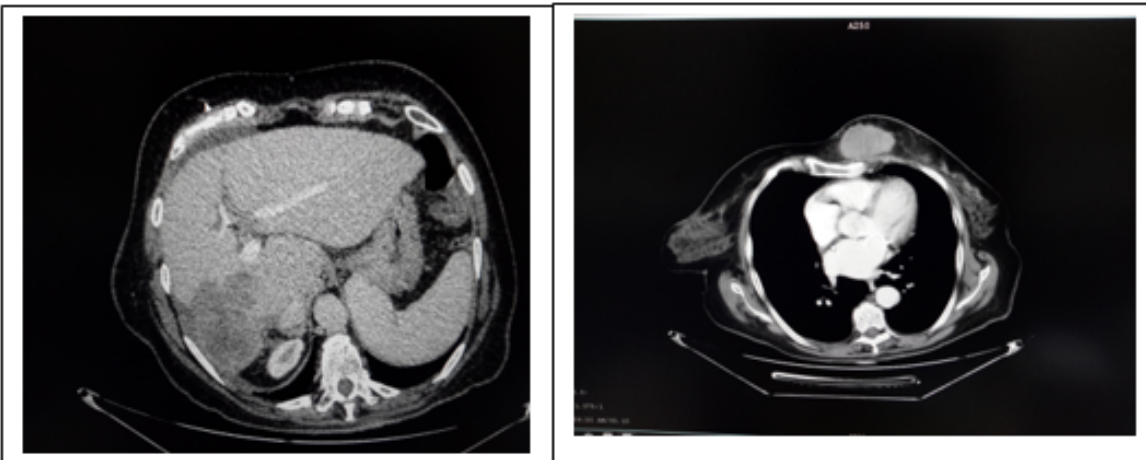

Figure 1: Chest and abdominal CT scan showing a left breast mass with irregular borders and a mass $7.9 \times 8.3 \mathrm{~cm}$ on the VII liver segment, with enlarged hepatic lymph nodes and splenomegaly. 
A 70 years old Caucasian female, with chronic HCV infection, presented on the outpatient clinic, during routine follow-up after right hepatectomy, complaining of mastalgia and a palpable mass on her left breast, discovered during self-palpation. The mass was rigid, tender about $5 \times 4 \mathrm{~cm}$ in size. She had a previous surgical history of right hepatectomy 8 months ago, due to hepatocellular carcinoma in segments VI, VII of the liver, after an embolism of the right portal vein and counterbalancing hypertrophy of the left liver lobe. The biopsy had reported hepatocellular carcinoma with neuroendocrine differentiation, grade III-IV Edmondson-Steiner, CK8-, CK18+, CK7+, hepatocyte+, glypican-3+, AFP+, pCEA+, $\beta$-catenin,$+ \mathrm{s}-100-$, CHR moderately positive, SYN moderately positive, CD56-, Ki 67 80\% . During surgical follow- up, the patient underwent CT scan of the abdomen, thorax and brain, which showed a left breast mass with irregular borders $5.9 \times 4.5 \mathrm{~cm}$ and a mass $7.9 \times 8.3 \mathrm{~cm}$ on the VII liver segment, with enlarged hepatic lymph nodes and splenomegaly (Figure1).

The mammogram showed a non-homogenous mass on the inner side of the left breast, below the papilla, with size $4 \times 3 \times 2.4 \mathrm{~cm}$. with irregular borders (Figure 2). Afterwards, the patient underwent an FNA biopsy, which showed malignant cells, most probably originating from adenocarcinoma. Serum cancer biomarkers AFP, Ca125 and Ca 15-3 were increased. After an MDT discussion, the patient underwent a trans arterial chemoembolization of the HCC recurrence and afterwards, a left mastectomy. The pathologic examination of the left breast reported a neoplastic mass with characters of metastatic hepatocellular Ca with neuroendocrine differentiation, histological scale of III-IV Edmondson-Steiner, CK8/18(+), Hepatocyte(+), Glypican-3(+), aFP(+), Arginase-1(),Synaptophysin (+) locally, Chromogranin (+) locally CD56(-), GATA$3(-), E R(-)$, PR(-), Ki67 88\%. The patient was discharged home, the third postoperative day, in good clinical condition, but according to our follow-up, died from disease progression six months, after the mastectomy.

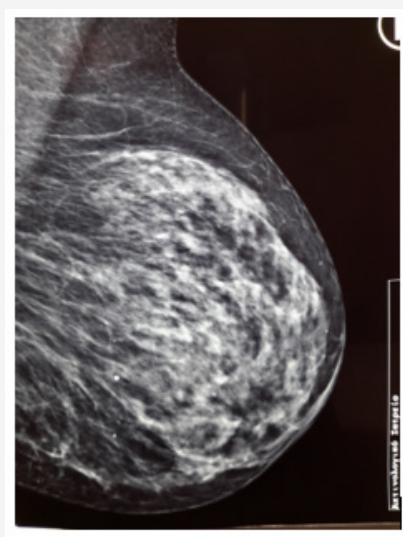

Figure 2: Mammogram of the left breast showing a nonhomogenous mass on the inner side of the left breast, below the papilla, with irregular borders.

\section{Discussion}

The incidence of hepatocellular carcinoma varies among geographical areas, with greater incidence being found in Asia,
Taiwan, Korea, China and Japan [1-7]. Advanced hepatocellular carcinoma is one of the most lethal diseases and includes metastases to various organs, such as lungs, lymph nodes, musculoskeletal structures, peritoneal surface, gastrointestinal tract, spleen and pancreas [7-10]. It has only few systematic therapeutic options, with Sorafenib, being the most important pos administered molecular drug, that increases overall survival by approximately 3 months (10.7 months) compared with placebo (7.7 months) [6].

To the best of our knowledge, this is the second case report of a breast metastasis from primary hepatocellular carcinoma. Lo et al, reported three cases of breast metastases in three patients with disseminated hepatocellular carcinoma. The only difference is the fact that our patient had only breast metastasis whereas Lo et al patients had disseminated disease [1]. Musculoskeletal metastases from hepatocellular carcinoma accounts for approximately $20 \%$ of metastatic lesions. Kim et al, reported scapular metastasis with intramuscular hematoma from primary hepatocellular carcinoma, in a patient with back pain [2]. Bachtiar et al, reported right mandible and coracoid process metastases, in a patient with newly diagnosed hepatocellular carcinoma, that had right lower tooth pain and swelling over the right mandible, as well as right shoulder pain, with no associated trauma [7]. Maharajan et al, reported a L3 vertebra solitary metastasis, from primary clear cell carcinoma of the liver, presenting as low back pain and Adnot et al, published a case report, describing a maxillary metastasis, presenting as a hemorrhagic mass of the oral cavity $[8,9]$.

Al-Sharidah et al, reported a case of a 31 years old female patient, with chronic HBV infection, that presented to the outpatient clinic with 6 months mastalgia, and a palpable mass on the left breast. In contrast to our case, though, it was revealed through CT scan of the thorax, that the mass had origin on the 3rd rib of the left thorax and was bordering the ipsilateral major pectoralis muscle, invading the muscle and the soft tissue of the breast. The patient underwent an image-guided needle biopsy of the mass, that confirmed a metastatic epithelial tumor of hepatic origin [5]. Moreover, Patel et al, reported a cutaneous metastasis on the left lateral eyebrow, as the first clinical presentation of hepatocellular carcinoma, whereas, Greco et al, reported a cardiac metastasis in a patient with known hepatocellular carcinoma and hypertrophic cardiomyopathy, that presented in the emergency department, with syncope-like episode. MRI and CT scan, showed abnormal thickening of the interventricular septum, extending to the entire apex and the anterior and lower segments of the left ventricle. A biopsy was conducted, confirming the diagnosis of cardiac infiltration from hepatocellular carcinoma $[3,4]$.

Harada et al, reported a case of asymptomatic oesophageal metastasis, found on routine basis, in a patient with hepatocellular carcinoma, that underwent an oesophagectomy with thoracotomy and the biopsy showed metastasis from hepatocellular carcinoma, whereas, Salimon et al, reported a case of symptomatic oesophageal metastasis in a patient with hepatocholangiocarcinoma and dysphagia. The patient was treated with chemotherapy, contrary 
with the previous one $[6,10]$. Nugroho and Serra et al, reported cases of adrenal gland metastases from primary hepatocellular carcinoma, diagnosed with CT-guided biopsy, that were treated with chemotherapy $[11,12]$ whereas, Zhigui Li et al, reported a case of a patient with multiple ectopic liver tissues along the pancreas, with development of hepatocellular carcinoma on these [15]. Furthermore, Yeh et al, reported a case of hepatocellular carcinoma metastasis to the left kidney, whereas Kanazawa et al, reported a case of jejunal metastases with primary hepatocellular carcinoma, that presented with melena and anaemia $[16,17]$.

Qianhui Li et al, published a case of a 59 years old patient with cryptorchidism and a right inguinal mass along with a liver mass, who underwent a right orchidectomy whose biopsy, spoke of a metastasis from primary hepatocellular carcinoma [13] Finally, Morais et al, reported a case of sphenoid and cavernous sinuses metastases, in a patient with hepatocellular carcinoma, complaining of headache and photophobia and Yen et al, presented a case of mediastinal, lung and pleural metastases with associated hemothorax, in a patient with hepatocellular carcinoma, that entered the hospital for chemotherapy $[18,19]$.

\section{Conclusion}

Breast metastases from hepatocellular carcinoma are extremely rare and to the best of our knowledge, this is the second case report worldwide, describing this clinical entity, that needs significant clinical suspicion to be diagnosed.

\section{Acknowledgement}

None.

\section{Conflict of Interest}

No conflict of interest.

\section{References}

1. Lo HC, Lee KF, Yeh CN, Chen MF (2004) Breast metastasis from hepatocellular carcinoma. J Hepatogastroenterology 51(56): 387-390.

2. Kim KH, Oh HH, Son DJ, Hong JY, Jeong YH, et al. (2017) Scapular metastasis of hepatocellular carcinoma presenting as acute bleeding and hematoma. A case report of safe and effective treatment. Medicine (Baltimore) 96(46): e8736.

3. Patel N, Sheehan Dare G, Weir J, Verma S, Fearfield L (2018) Cutaneous metastasis as the first presentation of hepatocellular carcinoma. Hepatology 67(4):1631-1633.

4. Greco A, De Masi R, Orlando S, Metrangolo A, Zecca V, et al. (2017) Metastases of hepatocellular carcinoma misdiagnosed as isolated hypertrophic cardiomyopathy. Ann Hepatol16(6): 966-969.
5. Al Sharydah AM, Al Abdulwahhab AH, Alghnimi IA, El Shawarby MA, Katbi FA (2017) Mastalgia as an atypical presentation of hepatocellular carcinoma: a case report. World J Surg Oncol 15(1): 58.

6. Harada JI, Matsutani T, Hagiwara N, Kawano Y, Matsuda A, et al. (2018) Metastasis of hepatocellular carcinoma to the esophagus: case report and review. Case Rep Surg.

7. Md Radzi AB, Tan SS (2018) A case report of metastatic hepatocellular carcinoma in the mandible and coracoid process. A rare presentation. Medicine (Baltimore) 97(4): e8884.

8. Maharajan K, Hey HWD, Tham I, Thamboo TP, Wong A, et al. (2017) Solitary vertebral metastasis of primary clear cell carcinoma of the liver: a case report and review of literature. J Spine Surg 3(2): 287-293.

9. Adnot J, Gemival P, Trost 0 (2018) Maxillary metastasis from a hepatocellular carcinoma: Report of an uncommon presentation and literature review. J Stomatol Oral Maxillofac Surg 119(6): 503-505.

10. Salimon M, Chapelle N, Matysiak Budnik T, Mosnier JF, Frampas E (2018) et al. Esophageal metastasis of stem cell-subtype hepatocholangiocarcinoma: rare presentation of a rare tumor. World J Gastroenterol 24(7): 870-875.

11. Nugroho A, Lee KW, Lee KB, Kim HS, Kim H, et al. (2018) Adrenal metastasis in sequentially developed combined hepatocellular carcinoma-cholangiocarcinoma: a case report. Ann Hepatobiliary Pancreat Surg 22 (3):287-291.

12. Serra K, Llatjos R, Catalá I, Gornals JB (2017) Adrenal metastasis of hepatocellular carcinoma diagnosed by endoscopic ultrasound-guided fine-needle aspiration of the right adrenal gland. Rev Esp Enferm Dig 109(5): 378.

13. Qianhui Li, Xiuying Shi, Chuifeng Fan (2018) A metastasized hepatocellular carcinoma in the capsule of an undescended testis in the right inguinal area: report of a rare case. World J Surg Oncol 16(1): 12.

14. Kotsakis A, Ardavanis A, Koumakis G, Samantas E, Psyrri A, et al. (2019) Epidemiological characteristics, clinical outcomes and management patterns of metastatic breast cancer patients in routine clinical care settings of Greece: Results from the EMERGE multicenter retrospective chart review study. BMC Cancer 19(1): 88.

15. Li Z, Wu X, Wen T, Li C, Peng W (2017) Multiple ectopic hepatocellular carcinomas in the pancreas: A case report Medicine (Baltimore) 96(30): e6747.

16. Tyng Yuan Jang, Ming Lun Yeh (2017) Hepatocellular carcinoma metastatic to kidney mimicking renal cell carcinoma. Kaohsiung J Med Sci 33(3): 161-162

17. Kanazawa M, Sugaya T, Tominaga $\mathrm{K}$, Takahashi $\mathrm{F}$, Takenaka $\mathrm{K}$, et al. (2018) Jejunal metastasis from hepatocellular carcinoma. Intern Med 57 (7): 947-950.

18. Rui Morais, Helder Cardoso, Marco Silva, Macedo G (2018) Hepatocellular carcinoma metastasis to sphenoid and cavernous sinus: An unexpected cause of ptosis. Dig Liver Dis 50(1): 95.

19. Yen CW, Hsu LS, Chen CW, Lin WH (2018) Hepatocellular carcinoma with thoracic metastases presenting as hemothorax: a case report and literature review. Medicine (Baltimore) 97(22): e10945. 